Meta

Journal des traducteurs

Translators' Journal

\title{
La gestion de la documentation au Bureau des traductions du gouvernement du Canada
}

\section{Suzanne Richer}

Volume 25, numéro 1, mars 1980

La documentation

URI : https://id.erudit.org/iderudit/003950ar

DOI : https://doi.org/10.7202/003950ar

Aller au sommaire du numéro

Éditeur(s)

Les Presses de l'Université de Montréal

ISSN

0026-0452 (imprimé)

1492-1421 (numérique)

Découvrir la revue

Citer cet article

Richer, S. (1980). La gestion de la documentation au Bureau des traductions du gouvernement du Canada. Meta, 25(1), 49-57. https://doi.org/10.7202/003950ar d'utilisation que vous pouvez consulter en ligne. 


\title{
La gestion de la documentation au bureau des traductions du gouvernement du Canada
}

\author{
SUZANNE RICHER \\ directeur de la documentation, \\ Bureau des traductions, Ottawa
}

La chaîne documentaire se définit souvent comme étant le passage de l'information d'une mémoire à une autre. Ce processus d'extraction, de saisie, de traitement et de diffusion des données est plus ou moins raffiné. Sur le plan international, le NATIS ou système national d'information est ainsi défini dans les documents de la Conférence intergouvernementale sur la planification des infrastructures nationales en matière de documentation, de bibliothèques et d'archives de l'Unesco (Paris, 1974) : «Les éléments qui doivent entrer dans la composition du NATIS sont tous les services qui contribuent à apporter de l'information à tous les secteurs de la communauté et à toutes les catégories d'usagers. La tâche du NATIS est d'assurer que tous ceux qui sont engagés dans des activités politiques, économiques, scientifiques, éducatives, sociales ou culturelles reçoivent l'information nécessaire, qui leur permettra d'apporter leur contribution maximale à la communauté dans son ensemble ${ }^{1}$. »

On peut aussi parler de réseaux de services d'information nationaux spécialisés tel le Service d'information technique (SIT) du Conseil national de recherches du Canada dont les «objectifs sont d'améliorer la technologie de la production industrielle, d'optimiser l'utilisation industrielle des ressources financières, humaines et matérielles, de faire connaître et de rendre accessible à l'industrie l'information scientifique et technique dont elle a besoin ${ }^{2} 》$.

Et finalement, on en arrive aux modules de services documentaires qui fonctionnent selon les mêmes principes directeurs mais dans des cadres beaucoup plus réduits. Il n'en reste pas moins que les réalités essentielles de la chaîne documentaire sont toujours les mêmes; les constantes du concept qu'il soit international, national ou local sont donc : 1) l'information, 2) l'usager et 3) l'intermédiaire.

L'information est souvent habillée, traitée ou diluée dans des réalités physiques appelées collections, banques de données, bibliothèques, toutes, indépendamment de leur forme, des outils ou des coffrets développés pour la conservation de l'information. Donc, difficulté ou encore danger pour l'usager non initié de ne

1. Systèmes nationaux d'information (NATIS), Paris, Unesco, 1974 (com. 74/NATIS/3,

2. Canada, Conseil national de recherches, Rapport du président 1975-1976, Ottawa, 1976, p. 91. 
pouvoir extraire l'information voulue ou le détail spécifique recherché. D'où le rôle prépondérant de l'intermédiaire spécialisé dans les techniques de l'information; le bibliothécaire ou documentaliste acquiert, traite, organise et diffuse les données bibliographiques et documentaires.

La réalité « information/usager» de même que la réalité «information/ bibliothécaire »sont essentiellement directes tandis que la relation « usager/bibliothécaire » est artificielle en ce sens qu'elle est obligatoirement mise en place afin de faciliter l'accès à l'information pour l'usager qui est dans l'impossibilité de se tenir au courant des facteurs de normalisation bibliographique développés en fonction de l'exploitation des systèmes documentaires. Par conséquent, le bibliothécaire ne doit jamais renverser les rôles et toujours se placer dans son cadre de fonctionnement c'est-à-dire se définir essentiellement comme un lien entre l'information et l'usager. Le rôle du bibliothécaire a cependant plusieurs facettes : intermédiaire entre les unités de travail et les centres d'information dépositaires de ressources documentaires, conseiller en recherche documentaire et en matière de développement des collections et même un rôle pédagogique i.e. responsable de la formation documentaire des usagers de son service de bibliothèque.

Toute la philosophie du service de la Direction de la Documentation du Bureau des traductions repose et évolue autour des composantes « information/ usager/bibliothécaire »et de la relativité qui régit cet équilibre.

Jusqu'au moment de l'engagement d'un bibliothécaire professionnel en 1974, les documents acquis et consultés par les traducteurs et les terminologues du Bureau étaient gracieusement et louablement organisés par des traducteurs dont la rigueur méthodique et l'esprit universel étaient exceptionnels. Le travail de conservation et d'indexation du matériel documentaire entrepris par ces érudits a jeté la base des services de bibliothèque actuels. De 1974 à 1977, le besoin d'un service de bibliothèque géré professionnellement est né, s'est développé et s'est transformé en réalité.

La création de la Direction de la Documentation en juin 1977, au moment même où les services de bibliothèque du Bureau connaissaient un changement de directeur, concrétisa la nouvelle philosophie des services documentaires du Bureau. L'étude de la clientèle entreprise par la nouvelle équipe de gestionnaires permit d'identifier immédiatement les paramètres du service à donner. Les éléments distinctifs de même que les dénominateurs communs entre les trois éléments du Bureau à savoir traducteurs, terminologues et interprètes ont été décelés, analysés et interprétés en fonction du choix d'un modèle documentaire capable de répondre aux besoins des usagers, besoins quelquefois exprimés clairement et parfois besoins latents.

Un plan quinquennal en matière de service documentaire a donc été établi en février 1978 ; ce plan-cadre déterminait les besoins actuels de la clientèle, les ressources humaines, financières et documentaires dont la Direction de la Documentation disposait et proposait finalement un programme pour les années 1978-1983. Les ressources humaines nécessaires aux réalisations professionnelles et techniques ont été identifiées, les modalités et les activités du service de 
référence et du traitement de la documentation ont été analysées et la structure du réseau de bibliothèques et de modules documentaires a été arrêtée. Le modèle documentaire choisi est plutôt conventionnel puisqu'il est basé sur un ensemble de petites bibliothèques locales (bibliothèques de sections de traduction et de terminologie) appuyés par 4 bibliothèques dites « de réseau » appelées à compléter les collections des bibliothèques de section et à dispenser des services de référence ponctuelle et thématique, de prêts entre bibliothèques, de choix de collection. Cet ensemble de modules documentaires est voué à la communication avec l'usager, à la diffusion de l'information et à l'analyse constante des besoins locaux.

Des services techniques centralisés aux Terrasses de la Chaudière à Hull se chargent des acquisitions de matériel pour l'ensemble du réseau, du traitement de la documentation (catalogage, classification et codage de sources) tandis que des services de référence offrent un éventail d'activités documentaires aux bibliothèques du réseau en mettant à leur disposition des spécialistes du SVP, de la référence bibliographique et automatisée, du prêt entre bibliothèques et du développement des collections.

La structure que s'est donnée la Direction de la Documentation pour atteindre son objectif de service compte trois divisions : la Division des services publics, la Division des services techniques et la Division de la coordination du réseau de bibliothèques. Les trois divisions sont sous la direction de bibliothécaires professionnels responsables des opérations techniques et professionnelles de leur secteur d'activité, de la coordination de leurs activités avec les autres divisions et finalement de la gestion des ressources humaines qui leur sont allouées. Le catalogue collectif ou inventaire des ressources documentaires du Bureau est le centre nerveux de cet appareil documentaire; la centralisation des acquisitions et du traitement catalographique favorise la tenue d'un catalogue collectif. La Direction de la Documentation utilise les services informatiques d'UTLAS (University of Toronto Library Automation Systems) pour des fins de traitement catalographique, d'automatisation des codes de source, de production de listes d'acquisition et éventuellement de produits micrographiques tel le fichier collectif sur COM. La Direction de la Documentation dépense quelque $\$ 200000$ par année pour acheter de la documentation afin de répondre tant soit peu aux besoins de la clientèle; les sommes paraissent énormes, mais n'oublions pas que les ouvrages de référence sont les plus coûteux selon les statistiques du marché canadien du livre et que la dispersion des effectifs du Bureau des traductions à travers le Canada exige des collections locales de référence répondant aux besoins essentiels et primaires des sections de traduction et de terminologie. La politique de développement et de rationalisation des collections de la Direction permet de profiler les besoins des sections et de catégoriser ces besoins; il est ainsi possible d'éviter de répéter des collections ou des titres coûteux puisque l'index des profils documentaires identifie les intérêts de chaque section et par conséquent ses ressources documentaires. Le catalogue collectif est un autre élément de rationalisation des collections puisqu'il favorise le prêt entre bibliothèques à l'intérieur du réseau de bibliothèques du Bureau; de plus, la gestion de nos données bibliographiques par UTLAS permet l'accès à une banque de données importante puisque 
plus de 90 bibliothèques et réseaux de bibliothèques participent à UTLAS qui offre une banque de données de quelque 6 millions de notices bibliographiques dont 11/2 million proviennent des fichiers-sources tels le LC/MARC de la Library of Congress, le CAN/MARC de la Bibliothèque nationale du Canada, le Fichier MARC/Québécois de la Bibliothèque nationale du Québec, etc. ${ }^{3}$ Là encore, le prêt entre bibliothèques est facilité par l'utilisation de cette banque de données pour des fins de référence par le biais de REFCATSS. UTLAS nous permet aussi de manipuler nos dossiers bibliographiques pour en extraire des listes de vocabulaires, dictionnaires, un répertoire de codes de source, etc.

\section{LA DIVISION DES SERVICES TECHNIQUES}

La Division des services techniques comprend une Section d'acquisition (monographies, périodiques, publications en série, etc.) et une Section de catalogage. L'utilisation de la politique de développement des collections par les sections de traduction et de terminologie complétée par la rationalisation des demandes de documents faite par les bibliothèques de réseau et la Section du développement des collections permet de tirer le maximum des fonds dont dispose la Direction de la Documentation. La Section des acquisitions répond à plus de 7000 demandes de documents par année en provenance des quelque 140 unités de travail du Bureau réparties à travers le Canada. De plus, les Acquisitions assurent l'abonnement, le réabonnement et la réclamation des livraisons manquantes pour les 1000 abonnements à plus de 720 titres. Une liste des publications en série répertoriant les titres reçus avec mention de code de localisation est en voie de préparation. La Division des services techniques étudie actuellement la possibilité d'automatiser d'ici 2 ou 3 ans les acquisitions de monographies et de publications en série. D'ici quelques années d'ailleurs, UTLAS aura développé les modules nécessaires et une telle approche permettra à la Direction de développer un système intégré pour le traitement de la documentation du Bureau.

La Section du catalogage offrira d'ici quelques mois aux usagers du Bureau un catalogue sur microfiche des ressources documentaires du Bureau des traductions en utilisant la banque de données bibliographiques et les ressources informatiques d'UTLAS. Les ouvrages répertoriés au catalogue comprendront les nouvelles acquisitions reçues au cours de l'année, les ouvrages dont les notices bibliographiques auront été converties du fichier manuel et les ouvrages catalogués dans les collections existantes qui n'avaient jamais été répertoriées. Les sous-produits suivants pourront être offerts à partir de la banque de données automatisée : une liste hebdomadaire et cumulative par ordre de titres des nouvelles acquisitions, une liste mensuelle par ordre topographique des nouvelles acquisitions, un inventaire annuel de la documentation cataloguée pour chacun des emplacements du réseau.

Au chapitre du codage des sources, la Direction publiera et diffusera les résultats de son étude sur le code de source commencée en 1978. La Section

3. Jack Cain. Aperçu sur un grand réseau automatisé de bibliothèques en pleine croissance, Toronto, UTLAS, 1978, $11 \mathrm{p}$. 
fournit un service de codage systématique de tous les ouvrages catalogués par la Division des services techniques, de codage sur demande des titres et de décodage des codes soumis par les terminologues, les traducteurs et les pigistes du Bureau de même que par les autres organismes utilisant ou contribuant à la Banque de terminologie. Ce catalogue sera disponible sur support papier ou encore sur support micrographique. Les bandes magnétiques de ce catalogue seront mises à la disposition de la Banque de terminologie afin qu'elle puisse offrir en mode conversationnel les notices bibliographiques correspondant aux codes de source utilisés par la Banque pour citer les sources de la terminologie emmagasinée.

\section{LA DIVISION DES SERVICES PUBLICS}

La Division des services publics offire des services spécialisés de référence à tous les traducteurs, terminologues et interprètes du Bureau. Ses composantes sont : une Section de développement des collections et une Section de référence.

Le mandat de la Section du développement des collections est de développer les ressources documentaires du Bureau conformément aux profils d'intérêt des sections de traduction et de terminologie et des bibliothèques du réseau. On implante graduellement des mécanismes permettant d'accomplir un travail de sélection rationnel de la documentation de même que des modalités de contrôle des achats de volumes et abonnements de périodiques tout en offrant, le cas échéant, des possibilités tels le prêt et le prêt entre bibliothèques pour répondre aux besoins de la clientèle. Une étude est en cours pour déterminer si la Direction de la Documentation doit s'abonner au module NEPAL (New Publications Awareness List) d'UTLAS afin de réduire le temps passé au dépouillement des catalogues d'éditeurs et de répertoires bibliographiques ; on pourrait ainsi réduire le nombre d'outils de travail servant uniquement pour la sélection d'ouvrages.

Des «Listes de publications disponibles》 (gratuitement) sont compilées régulièrement et distribuées aux sections du Bureau et aux bibliothèques du réseau afin de compenser un budget d'achat qui diminue avec les années si l'on tient compte $d u$ coût des volumes qui augmente sans cesse.

A la Section de référence, le fichier des bibliographies compilées augmente au rythme d'une centaine par année ; chaque bibliographie est classée numériquement et l'accès se fait par vedettes-matières. On publie aussi des bibliographies dans le cadre de la collection DIC/VOC. En référence générale et au SVP documentaire, on participe à l'alimentation de la Banque de terminologie en y versant les mini-fiches remplies suite à des questions de traduction d'appellations officielles qui proviennent des traducteurs et terminologues. La Division des services publics a établi l'arbre des domaines pour les appellations officielles qui constitue la classe W des codes de domaines BTUM afin de classer et d'emmagasiner ces fiches dans la Banque; la Division est responsable de l'uniformité des données versées dans le fichier $\mathrm{W}$ de la Banque de terminologie du Bureau.

Dans le cadre d'un service de diffusion sélective de l'information (DSI), la Section de référence exploite les banques de données du système SABINE (Système Automatisé de Banques d'Information Non Numériques) offrant des 
références bibliographiques d'origine mondiale accessibles directement en langue française. On y dépouille également RADAR (Répertoire analytique des articles de revues du Québec). Nous sommes ainsi en mesure de fournir à notre clientèle de la documentation très récente et en langue française.

On utilise également le module REFCATSS (Reference Catalogue Support System) d'UTLAS pour compiler des bibliographies; ce module offre un accès par auteurs, titres, matières et mots clés. En différé, on fait appel aux services de CAN/SDI et CAN/OLE via la Bibliothèque nationale du Canada et l'Institut canadien pour l'information scientifique et technique (ICIST).

Dans le cadre d'un projet de dépouillement de revues de pointe, la Division des services publics propose dans l'établissement de la méthode de travail, de commencer à dépouiller un sujet d'intérêt particulier signalé par les comités de bibliothèque locaux tout en faisant une recherche rétrospective et en établissant un profil CAN/SDI ; cette conjugaison d'activités permettra aux traducteurs et terminologues intéressés par le sujet en question de recevoir périodiquement les nouveautés bibliographiques. De plus, la Section de référence dépouillera un certain nombre de revues et périodiques reçus par le Bureau des traductions et publiera sous forme de \&bulletin signalétique» les résultats de ce travail de dépouillement.

L'organisation, le classement et la diffusion de dossiers-documentation est une autre activité de référence. On prévoit faire, d'ici quelque temps, un travail d'uniformisation des dossiers-documentation dans les différentes bibliothèques du réseau afin de rendre ces documents accessibles à l'ensemble de notre clientèle.

La demande de prêts entre bibliothèques est plutôt stable et se situe aux environs de quelque 200 demandes par mois. L'utilisation de REFCATSS permet de localiser rapidement un ouvrage dans les grandes bibliothèques canadiennes, le LDS (Library Delivery Service) de la Bibliothèque nationale du Canada est beaucoup plus efficace que la poste pour le transport du matériel documentaire emprunté et prêté et enfin l'utilisation du Télex pour obtenir rapidement certains prêts à l'extérieur de la région de la capitale nationale sont tous des moyens employés en vue de réduire les délais de localisation et d'envoi du matériel demandé.

\section{LA DIVISION DE LA COORDINATION DU RÉSEAU DE BIBLIOTHÈQUES}

Cette Division gère les activités professionnelles et administratives des bibliothèques du réseau dont le dénominateur commun est évidemment l'excellence du service offert à la clientèle. Certains paramètres fondamentaux différencient entre elles ces bibliothèques : caractéristiques de la clientèle à servir, ressources documentaires disponibles.

Les grandes bibliothèques de réseaux sont :

1) La bibliothèque de la Direction générale des services de traduction (DGST)

Logée au $5^{\mathrm{e}}$ étage des Terrasses de la Chaudière à Hull, cette bibliothèque coordonne les services documentaires dispensés à quelque 53 sections de traduc- 
tion réparties dans divers édifices de la région de la capitale nationale. Ses ressources documentaires comprennent une collection générale, une documentation spécialisée dans les domaines scientifique et technique, des ouvrages de référence pouvant soutenir le travail traductionnel d'une équipe de spécialistes multilingues de même que des collections propres au domaine militaire. La bibliothèque de la DGST a plusieurs succursales toutes dans la région d'Ottawa-Hull dont deux, soit la bibliothèque scientifique et technique et la bibliothèque multilingue, sont logées aux Terrasses de la Chaudière.

Cette bibliothèque est sous la direction d'une bibliothécaire professionnelle qui assure avec son équipe de bibliotechniciens et de commis les services de référence bibliographique et de type SVP, de consultation et de prêts entre bibliothèques de même que de développement des collections. Les services spécialisés et élargis offerts par la Division des services publics complètent le mandat de référence des bibliothèques de réseau. Le rôle du bibliothécaire en est un de communication et de formation; il est essentiel que la liaison « usager/bibliothécaire » soit saine pour qu'un climat de confiance s'établisse entre les deux parties. Le bibliothécaire est aussi secrétaire du comité local de bibliothèque ; par le biais de ce mécanisme, il peut aider à la formation de ses usagers en plus d'être à leur écoute et de leur transmettre les modalités de la philosophie documentaire de la Direction.

\section{2) La bibliothèque de la Division de Montréal}

Ce module documentaire dessert quelque 160 traducteurs et terminologues dont les besoins de référence et de collection sont très généraux. La collection de la bibliothèque offre une variété de documents (périodiques et monographies) dans les diverses disciplines de travail des usagers. Les collections des bibliothèques de section sont évidemment plus spécialisées en ce sens qu'elles répondent aux besoins précis d'une équipe de traducteurs tandis que la collection de la bibliothèque en est une de référence générale permettant de compléter les ressources documentaires spécialisées.

Cette bibliothèque offre donc des services de référence bibliographique et de type SVP, de prêts entre bibliothèques et de développement des collections.

\section{3) La bibliothèque de la Division de Québec}

Cette bibliothèque a une clientèle de quelque 90 traducteurs et terminologues en plus des deux sections d'Halifax et de Moncton qui utilisent ce module documentaire pour compléter leurs collections locales et obtenir des services de bibliothèque spécialisés. La collection de la bibliothèque de Québec est hautement spécialisée dans les domaines de l'art militaire et autres disciplines connexes ; cette bibliothèque est dépositaire de certaines publications des Forces armées canadiennes suite à une entente de principe, genre dépôt légal, avec les organismes militaires en question.

Le personnel documentaire offre des services de référence, de prêts entre bibliothèques et de développement des collections. Cette bibliothèque sert aussi de 
centre spécialisé en art militaire tout comme la nouvelle bibliothèque traductionterminologie au quartier général du ministère de la Défense nationale à Ottawa; cette dernière offre cependant le service de référence SVP à cause de la vocation de spécialisation de l'équipe de terminologues sur place. Ce SVP militaire complète les activités de référence du SVP général offert par la Division des services publics dans le cadre du service terminologique dispensé par le Bureau des traductions.

La bibliothèque de la Division de Québec coordonne les demandes d'achat des deux sections d'Halifax et de Moncton; cette bibliothèque agit aussi comme agent de liaison en matière de référence et de prêts entre bibliothèques. Elle communique avec les services centralisés de la Direction de la Documentation pour tous les cas qui concernent ces deux sections éloignées.

\section{4) Le Centre de documentation de la Direction générale de la terminologie et de la documentation (DGTD)}

La clientèle du centre de documentation est composée principalement de terminologues; ce module documentaire dessert cependant aussi des agents de liaison et de normalisation, des informaticiens et le personnel professionnel et technique de la Direction de la Documentation. La collection en est une de référence et de recherche, car elle répond aux besoins souvent opposés des «svpistes » et des thématiciens. Cela suppose donc une collection dont les modalités de contrôle du prêt entre bibliothèques et au comptoir doivent être régies selon une politique différente de celle appliquée dans le cas des bibliothèques desservant les sections de traduction. La documentation doit être disponible à tout instant pour permettre aux terminologues et bibliothécaires des services SVP de fournir les renseignements demandés et doit aussi être facilement consultable sur place pour les terminologues affectés à des recherches thématiques et pour les bibliothécaires responsables de la compilation de bibliographies. D'autre part, la collection est sujette à être empruntée par blocs importants si le terminologue doit travailler à long terme sur un sujet particulier. D'où le besoin d'avoir des exemplaires doubles de certains titres ou encore de retenir en réserve certains documents que ce soit des monographies ou encore des périodiques.

Le fonds documentaire du centre de documentation de la DGTD est composé d'ouvrages de référence tels des dictionnaires, encyclopédies, thésauri, vocabulaires, lexiques, guides et manuels, etc., fournissant une terminologie ou encore une possibilité d'extraction de terminologies plus ou moins spécialisées selon les disciplines. Le Centre de documentation possède aussi les collections complètes des normes en français et en anglais de l'ISO (Organisation internationale de normalisation) et de l'ONGC (Office des normes du gouvernement canadien), ainsi qu'une collection complète des normes de l'AFNOR (Association française de normalisation) ; il est évident que l'acquisition de telles collections comporte des responsabilités nationales tels le prêt entre bibliothèques et la consultation sur place de ces documents pour les usagers de la région de la capitale nationale. 
En plus de cette spécialisation des collections, le centre de documentation dispense des services de référence ponctuelle et thématique, de développement des collections et de prêt entre bibliothèques. Le volume de transactions en matière de circulation au comptoir est élevé puisque cette bibliothèque est le plus ancien module de l'actuel réseau de bibliothèques de la Direction de la Documentation et que la collection est évidemment plus développée et mieux adaptée aux besoins des usagers ; il appert aussi que la consultation sur place est fréquente et ceci est dû à l'essence même du fonds documentaire qui en est un d'ouvrages de référence.

Les bibliothèques du réseau sont le fondement de la relation « information/ usager $\gg$ tandis que le catalogue collectif en est l'instrument qui permet la diffusion de l'information stockée. L'équipe de communication formée de bibliothécaires et de bibliotechniciens n'a pas comme seule tâche la diffusion de l'information et de la documentation mais doit aussi générer de nouvelles formules documentaires, établir des mécanismes de dépistage des besoins des usagers et aussi créer des besoins chez l'usager afin de maintenir le dynamisme évolutif des services documentaires.

\section{CONCLUSION}

Le directeur de la Documentation rend compte des activités documentaires du Bureau à la Chambre intersectorielle, un groupe permanent de responsables qui regroupe les directions générales du Bureau en vue d'assurer une meilleure information réciproque et une collaboration plus intelligente. Son mandat a donc été élargi en juin dernier pour se lire comme suit : « La Chambre intersectorielle est, en particulier, l'organe de consultation entre la Direction de la Documentation et les secteurs opérationnels. Elle étudie les politiques et le budget de la DD (Direction de la Documentation), achemine les opinions et fait des recommandations sur le service fourni par la DD, informe la DD de tout nouveau programme de traduction, interprétation et terminologie et fait part de cette activité dans tout rapport annuel soumis au Comité de régie (du Bureau des traductions).»

La communication hiérarchique est donc assurée horizontalement par le biais des comités de bibliothèque locaux et verticalement par le mandat de la Chambre intersectorielle. Ainsi, la Direction de la Documentation est en mesure de jouer pleinement son rôle au sein du Bureau des traductions.

La gestion de la documentation au Bureau des traductions du Gouvernement canadien se fait donc selon un modèle documentaire organisationnel conventionnel. Les services dispensés se définissent selon les normes reconnues dans le monde des bibliothèques spécialisées et le traitement des collections est aussi formel que dans une bibliothèque universitaire, par exemple, tout en offrant un raffinement terminologique par l'indication du code de source. 